20 patients 11 were ASA I, 3 ASA II, 5 ASA III, and 1 ASA IV grade. 7, 6, 3, 4 of 20 patients had blood loss $<500 \mathrm{ml}$, $500-1000 \mathrm{ml}, 1000-1500 \mathrm{ml}$, and $>1500 \mathrm{ml}$ respectively. All patients received Tranexemic acid as prophylaxis for hemorrhage in a dose of $10 \mathrm{mg} / \mathrm{kg}$ bolus followed by $3 \mathrm{mg} / \mathrm{kg}$ infusion. Two patients were not given due to cardiac issues and had more blood loss than their counterparts. All patients were shifted to SICU for 24 hours. There were 7 patients with $<500 \mathrm{ml}$ blood loss were managed by fluid resuscitation, 6 patients with $500-100 \mathrm{ml}$ were managed by fluids alone and if $\mathrm{Hb}<10$ were given $1 \mathrm{PRBC}, 3$ patients with $1000-1500 \mathrm{ml}$ loss were given 3PRBC if $\mathrm{Hb}<10: 2 \mathrm{PRBC}$ if $\mathrm{Hb}>10$ and remaining four patients with $>1500 \mathrm{ml}$ loss were managed by three or more PRBC plus three or more FFPs. Conclusion: To conclude prophylactic administration of Tranexemic acid is helpful in decreasing the amount of bleed; however, the bleed will depend on the size and duration of surgery. With the above mentioned protocol we can achieve better hemodynamics intra-operatively and better post op $\mathrm{Hb}$.

\section{Intraoperative electro-oculographic monitoring to prevent postoperative extra-ocular motor nerve dysfunction during skull base surgeries}

\section{Veena Sheshadri, B . Suparna}

Department of Anaesthesia, Vikram Hospital, Bangalore, Karnataka, India

Background: To assess the feasibility of Electro-oculographic (EOG) monitoring in preventing extra-ocular motor nerve dysfunction during skull base surgeries. Materials and Methods: Intraoperative EOG recordings were obtained using disposable needle electrodes placed on the periorbital skin and the polarity of the waves noted for interpretation. The nerves were stimulated with a monopolar electrode during skull base exposure. Triggered as well as continuous EOG responses were recorded. Results: Of the nine cases monitored, oculomotor and abducent nerves were identified in all cases, but the trochlear nerve could not be definitively identified. Five out of nine patients had no pre or postoperative extra-ocular motor nerve dysfunction. The other four patients had pre-existing deficits before surgery, which recovered completely in two, significantly in one, and was unchanged in one patient at 3-6 month follow-up. Conclusion: EOG was found to be a simple and non-invasive method of monitoring extra-ocular motor nerves intra-operatively. Further studies in larger patient populations are required to quantitate the predictive value of EOG monitoring in detecting postoperative extra-ocular motor nerve deficits.

\section{Pharmacological and non- pharmacological interventions for reducing pain on rocuronium injection - A systematic review}

\author{
Bhavna Hooda, Hemanshu Prabhakar, Gyaninder Pal \\ Singh, Zulfiqar Ali ${ }^{1}$, Mani Kalaivani ${ }^{2}$ \\ Department of Neuroanaesthesiology, ${ }^{2}$ Biostatistics, AlIMS, \\ ${ }^{1}$ Anaesthesia and Intensive Care, Govt. Medical College, \\ Srinagar, India
}

Background: Various strategies, both pharmacological and non-pharmacological, have been studied to reduce the discomfort, incidence and severity of pain on rocuronium bromide injection. The objective of this review was to assess the ability of both pharmacological and non-pharmacological interventions designed to reduce or eliminate the pain that accompanies rocuronium bromide administration, both in adults and children, whether in asleep or awake state. Materials and Methods: We searched the Cochrane Central Register of Controlled Trials (CENTRAL), MEDLINE via Ovid SP (1966 to December 2012), and EMBASE via Ovid SP (1980 to December 2012). We included all randomized controlled trials (RCTs) that compared the use of any drug or a non-pharmacological method with control patients, or those receiving no treatment to reduce the severity of pain with rocuronium injection. Our primary outcome was pain on rocuronium bromide injection measured by a pain score assessment. Our secondary outcomes were rise in heart rate and blood pressure following administration of rocuronium and adverse events related to the interventions. We used the standardized methods for conducting a systematic review as described by the Cochrane handbook for Systematic Reviews of Interventions. Two authors independently extracted details of trial methodology and outcome data from reports of all trials considered eligible for inclusion. All analyses were made on intention-to-treat basis. We used a fixed-effect model where there was no evidence of significant heterogeneity between studies and a random-effects model if heterogeneity was likely. Results: We included 66 studies with 8621 participants in the review, though most analyses were based on data from fewer participants. We noted substantial heterogeneity between trials. Therefore, it was not possible to combine the trials for the outcomes. All studies reported the primary outcome pain; few studies reported adverse events and no study reported heart rate and blood pressure changes after administration of rocuronium. Conclusion: Lidocaine was the most popular intervention drug used in 29 trials with 2256 participants. The relative risk (RR) of pain on injection if given lignocaine compared to placebo was $0.21 ; 95 \%$ CI 
0.16 to 0.29 . Adverse events were reported mainly with use of opioids. They were cough, breath holding and chest tightness. The overall rate of complications was low. Both pharmacological and non-pharmacological methods were effective in reducing pain on injection of rocuronium. However, no one intervention can be considered superior over the other as the findings are not robust enough.

\section{Effect of intravenous dexmedetomidine in attenuation of hemodynamic response to intubation and skull-pin head holder application in patients undergoing elective neurosurgery}

\section{Amruta Ajgaonkar, Rajashree Gandhe}

Department of Anaesthesia, Kokilaben Dhirubhai Ambani Hospital and Medical Research Institute, Mumbai, Maharashtra, India

Background: The aim of this study is to determine the effect of intravenous dexmedetomidine in attenuation of hemodynamic response to intubation and skull-pin head holder application in patients undergoing elective neurosurgery. Materials and Methods: After approval from the hospital ethics committee, a prospective, randomized, double blinded study was conducted on 40 patients of ASA grades I or II, 18-65 years of age and undergoing elective neurosurgery. The intervention group received dexmedetomidine at $1 \mathrm{mcg} / \mathrm{kg} / \mathrm{hr}$ for 10 minutes followed by $0.5 \mathrm{mcg} / \mathrm{kg} / \mathrm{hr}$. The controls received normal saline at same rate. After standard induction, heart rate and mean arterial pressure were monitored at specified intervals during intubation and pin application. Intraoperative anesthetic and analgesic requirements were recorded. Complications, if any, were also noted. Appropriate statistical analysis was done. Data is presented as mean [SD]. Results: Demographics were comparable in both the groups. At one minute post-intubation, mean heart rate [hr] in control was 88.95 [15.05] and that in dexmedetomidine group 73.55 [11.70] $(P=0.001)$. The mean arterial pressure [MAP] in control was 105.35 [18.98] whereas that in dexmedetomidine group was 92.50 [4.38] $(P=0.020)$. On application of skull pin head holder, mean HR in control group was 93.2 [13.99] and 82.40 [11.18] in dexmedetomidine group $(P=0.01)$. MAP after application of skull pin head holder was 121.45 [SD 19.79] in control and 101.85 [SD 10.60] in dexmedetomidine group. Additional mean fentanyl requirement was $90 \mathrm{mcg}$ [49.74] in control group and $16 \mathrm{mcg}$ [34.70] in dexmedetomidine group $(P<0.00010)$. Conclusion: Intravenous dexmedetomidine is beneficial in attenuating the pressor response to intubation as well as application of skull pin head holder, without any serious adverse events. It also reduces the opioid and propofol requirement for the same.

\section{Comparison of oral intubation using flexible fibreoptic bronchoscope with or without rigid cervical collar: A clinical study}

Nitesh Gill, Shobha Purohit

Department of Anaesthesia, S M S Medical College, Jaipur, Rajasthan, India

Background: Device like fibreoptic bronchoscope is gold standard for difficult intubation situations. It can be performed by both orotracheal and nasotracheal route. But, through nasal route there are more chances of nasal bleeding and pressure necrosis while through oral route there is one problem which is clearing of upper airway for bronchoscope. Manoeuvres like jaw thrust and chin lift are very useful in clearing upper airway for bronchoscope. The aim of this study is to compare ease of oral intubation using flexible fibreoptic bronchoscope with or without rigid cervical collar, in terms of need of maneuvers: Jaw thrust and Chin lift. Materials and Methods: Twenty five patients in age range 20-50 years, of ASA I - $\Pi$, and of either sex undergoing elective surgery under general anesthesia were randomly allocated into each group. There were two groups: Group A (Rigid Cervical Collar) and group B (Without Collar). Intubating condition was assessed in between these groups with need of manoeuvres like jaw thrust and chin lift. Quantitative data is presented as mean values and standard deviation. Intergroup comparison of quantitative data was done by student's $t$-test and probability was considered to be significant if less than $<0.05$. Categorical data and need of manoeuvres (jaw thrust and chin lift) are presented as numbers and were compared among groups using Chi square test. $P<0.05$ was considered statistically significant. Results: There was

\begin{tabular}{lcccc}
\hline & $\begin{array}{c}\text { Group } \\
\text { A (with } \\
\text { collar) } \\
\boldsymbol{n = 2 5}\end{array}$ & $\begin{array}{c}\text { Group B } \\
\text { (without } \\
\text { collar) } \\
\boldsymbol{n = 2 5}\end{array}$ & Total & $\begin{array}{c}(\boldsymbol{P} \\
\text { value) }\end{array}$ \\
\hline $\begin{array}{l}\text { Need of } \\
\text { manoeuvres } \\
\text { (Jaw thrust and } \\
\text { chin lift) }\end{array}$ & 8 & 19 & 27 & 0.001 \\
$\begin{array}{l}\text { No need of } \\
\text { manoeuvres } \\
\begin{array}{l}\text { Jaw thrust and } \\
\text { chin lift) }\end{array}\end{array}$ & 17 & 6 & 23 & \\
Total & 25 & 25 & 50 & \\
\hline
\end{tabular}

